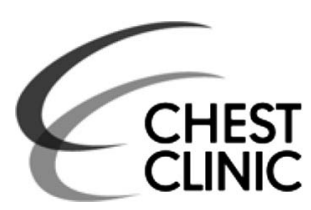

${ }^{1}$ Department of Medical Imaging, National Taiwan University Hospital, Taipei, Taiwan

${ }^{2}$ Department of Pathology, National Taiwan University Hospital, Taipei, Taiwan ${ }^{3}$ Department of Surgery, National Taiwan University Hospital and National Taiwan University College of Medicine Taipei, Taiwan

${ }^{4}$ Department of Traumatology, National Taiwan University Hospital, Taipei, Taiwan

${ }^{5}$ Department of Radiology, College of Medicine, National Taiwan University, Taipei, Taiwan

\section{Correspondence to} Yeun-Chung Chang, Department of Medical Imaging, National Taiwan University Hospital, No. 7, Chung-Shan South Road,

Taipei 100, Taiwan; ycc5566@ntu.edu.tw

Received 21 March 2015 Revised 5 October 2015 Accepted 19 October 2015 Published Online First 9 November 2015

\title{
An incidental pulmonary part-solid ground-glass nodule in a patient under anticoagulant therapy
}

\author{
Jo-Yu Chen, ${ }^{1}$ Huang-Chun Lien, ${ }^{2}$ Jin-Shing Chen, ${ }^{3,4}$ Yeun-Chung Chang ${ }^{1,5}$
}

\section{CASE PRESENTATION}

A 53-year-old man with a history of recurrent PE during 2008-2010, under long-term warfarin (Coumadin) therapy, had an incidental finding of a small subpleural part-solid ground-glass nodule (GGN) in the right upper lobe from a screening CT in December 2013 (figure 1A). The first follow up CT in April 2014 showed no significant interval change. The second follow-up CT in October 2014 showed enlargement of the part-solid GGN with pleural retraction (figure 1B), and malignancy was suspected. Video-assisted thoracoscopic wedge resection of the part-solid GGN was performed in November 2014.

\section{QUESTION}

What is your differential diagnosis for this partsolid GGN?

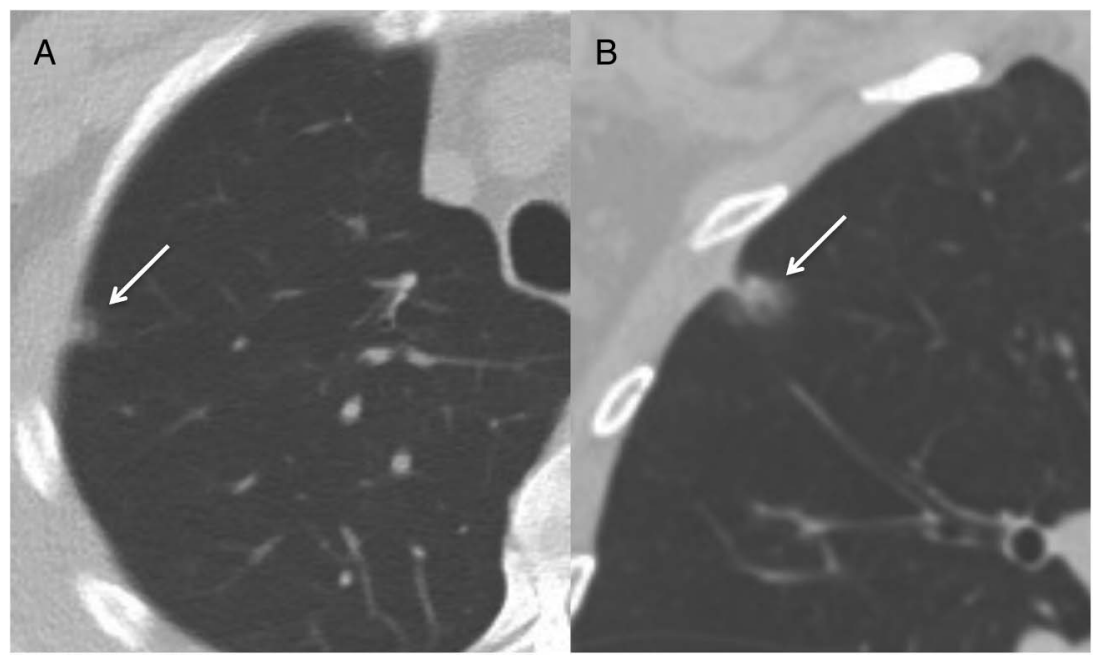

Figure 1 (A) CT in December 2013 on axial view showing a $10 \mathrm{~mm}$ subpleural part-solid ground-glass nodule (GGN) with solid component less than $5 \mathrm{~mm}$ in the right upper lobe. (B) Follow-up CT performed 10 months later on coronal view revealing enlargement of the part-solid GGN, about $18 \mathrm{~mm}$, with solid component about $8 \mathrm{~mm}$ and with pleural retraction. 


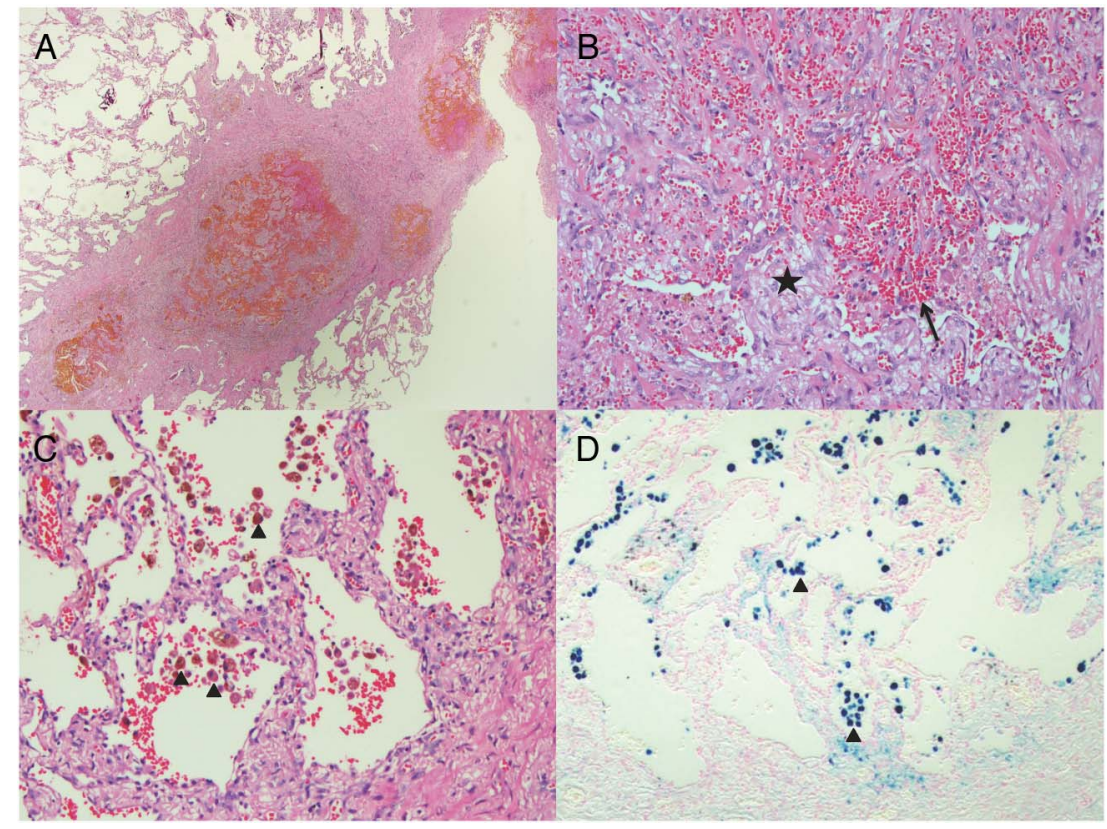

Figure 2 (A and B) The lesion features haphazardly arranged fibrotic tissue (star) mixed with haemorrhage (arrow). (C) Hemosiderin-laiden macrophage (arrowheads) can be seen within and surrounding the lesion. (D) Iron stain confirms the presence of hemosiderin (arrowheads).

\section{ANSWER}

The final pathological result revealed an organising haematoma with fibroinflammation.

As shown in figure $2 \mathrm{~A}, \mathrm{~B}$, the lesion features haphazardly arranged fibrotic tissue mixed with haemorrhage. Hemosiderin-laiden macrophage can also be seen within and surrounding the lesion (figure 2C, D) to indicate old haemorrhage. There is no definite evidence of coagulative necrosis of alveolar walls, vascular walls and bronchioles to suggest pulmonary infarction. There is also no arteriovenous malformation in the specimen.

The patient's prothrombin time international normalised ratio (PT INR) level was controlled between 1.07 and 2.60 during 2013-2014.

\section{DISCUSSION}

In this case, the enlarged part-solid GGN was presumed due to focal spontaneous pulmonary haemorrhage related to anticoagulant therapy. Pulmonary haematoma is an uncommon complication in patients with anticoagulant therapy. There were only two case reports of pulmonary haematoma secondary to anticoagulant therapy in the English literature. ${ }^{12}$ Both presented with large mass-like pulmonary haematoma. This case revealed a rare malignancy-mimicking part-solid GGN appearance of such complication.
The common differential diagnosis for solitary pulmonary GGN includes invasive adenocarcinoma, minimally invasive adenocarcinoma, adenocarcinoma in situ, atypical adenomatous hyperplasia and focal fibrosis. Part-solid GGNs have a greater likelihood of being malignant than pure GGNs. Persistent partsolid GGN with solid component larger than $5 \mathrm{~mm}$ or enlarged solitary part-solid GGN should be considered malignant until proved otherwise. ${ }^{3}$ Surgical intervention is indicated in our case.

Contributors J-YC wrote the manuscript, reviewed and reconstructed the images. $\mathrm{H}-\mathrm{CL}$ provided histopathology slides and descriptions. J-SC and Y-CC cared for the patient, did the intervention procedures, and critically revised the manuscript. All authors approved the final manuscript.

Competing interests None declared.

Patient consent Obtained.

Provenance and peer review Not commissioned; externally peer reviewed.

\section{REFERENCES}

1 Chakraborty AK, Dreisin RB. Pulmonary hematoma secondary to anticoagulant therapy. Ann Intern Med 1982;96:67-9.

2 Kaira K, Takei Y, Matsuura M, et al. Pulmonary hematoma resulting from anticoagulant therapy. AJR Am J Roentgenol 2003;180:1740.

3 Naidich DP, Bankier AA, MacMahon $\mathrm{H}$, et al. Recommendations for the management of subsolid pulmonary nodules detected at CT: a statement from the Fleischner Society. Radiology 2013;266:304-17. 